\title{
Highly efficient polarization control using subwavelength high contrast transmitarrays
}

\section{Amir Arbabi, Yu Horie, Mahmood Bagheri, Andrei Faraon}

Amir Arbabi, Yu Horie, Mahmood Bagheri, Andrei Faraon, "Highly efficient polarization control using subwavelength high contrast transmitarrays," Proc. SPIE 9372, High Contrast Metastructures IV, 93720H (27 February 2015); doi: 10.1117/12.2079559

SPIE. Event: SPIE OPTO, 2015, San Francisco, California, United States 


\title{
Highly efficient polarization control using subwavelength high contrast transmitarrays
}

\author{
Amir Arbabi ${ }^{1 *}$, Yu Horie ${ }^{1}$, Mahmood Bagheri ${ }^{2}$, Andrei Faraon ${ }^{1}$ \\ ${ }^{1}$ T. J. Watson Laboratory of Applied Physics, California Institute of Technology, 1200 E California \\ Blvd., Pasadena, CA 91125 \\ ${ }^{2}$ Jet Propulsion Laboratory, California Institute of Technology, Pasadena, CA 91109, USA \\ *amir@caltech.edu
}

\begin{abstract}
We report efficient wave plates with different retardations and orientations of fast axes realized using transmitarrays composed of a periodic arrangement of amorphous silicon elliptical cylinders on glass. We show that novel polarization devices which locally rotate the polarization by different angles while preserving the wavefront can be demonstrated using such a high contrast transmitarray. We present design, fabrication and experimental characterization results for near infrared transmissive wave retarders with efficiencies in excess of $90 \%$, and discuss the potential applications of atwill local polarization control enabled by this technology.

Keywords: Wave plate, metasurface, transmitarray, high index contrast, grating, cylindrical vector beams, radial and azimuthal polarizations, polarization control
\end{abstract}

\section{INTRODUCTION}

Light polarization is conventionally modified using wave plates made of birefringent materials such as crystalline quartz and polymer liquid crystals. Due to the small birefringence of these materials, the thickness of the wave plates is tens to hundreds of microns. Large birefringence may also be achieved using thin 1D or 2D dielectric and metallic gratings with subwavelength periods, and wave retarders have been implanted using these gratings [1-5]. The subwavelength grating based retarders not only provide polarization manipulation within a subwavelength thick layer but also allow for implementation of novel devices which can create a spatially varying polarization profile by locally modifying the polarization. These devices are created by gradually varying the grating parameters in the plane of the device, and realization of several devices have been reported using this technique $[2,6,7]$. It has also been shown that various phase masks for modifying wavefronts of circularly polarized beams can be realized by devices which function as half wave plates whose fast axis orientations are spatially varied $[7,8]$. These devices change the helicity of the incident circularly polarized beams while imposing spatially varying phase shifts which are referred to as geometric or Pancharatnam-Berry phase shits [9]. The majority of transmissive devices reported so far for local polarization modification or geometric phase based wavefront control, have low efficiencies or provide low spatial sampling resolution of the polarization and phase profiles $[6,10,11]$. Here, we present polarization control using an efficient high contrast transmitarray platform which also provides high resolution sampling of the desired polarization profiles. A high contrast transmitarray is a 2D array of potentially dissimilar high refractive index scatterers which are arranged on a periodic lattice with subwavelength lattice constant. The high refractive index contrast between the scatterers and their surrounding media leads to weak couplings among the scatterers, and allows for high resolution modification of light polarization by gradually changing the in-plane geometrical dimensions of the scatterers. We have recently shown that high contrast transmitarrays can be used to design highly efficient and polarization insensitive devices for high spatial resolution wavefront control $[12,13]$. Here, we show that novel polarization devices which locally rotate the polarization of the light by different angles while preserving its phase front can be realized using high contrast transmitarrays. We first present design, fabrication and experimental characterization results for near infrared wave retarders, and then for a device which generates cylindrical vector beams, and demonstrates the flexibility and the high efficiency provided by high contrast transmitarrays in polarization modification.

High Contrast Metastructures IV, edited by Connie J. Chang-Hasnain, David Fattal,

Fumio Koyama, Weimin Zhou, Proc. of SPIE Vol. 9372, 93720H · C 2015 SPIE

CCC code: $0277-786 \mathrm{X} / 15 / \$ 18 \cdot$ doi: $10.1117 / 12.2079559$

Proc. of SPIE Vol. $937293720 \mathrm{H}-1$ 


\section{HIGH CONTRAST TRANSMITARRRAY WAVE PLATES}

\subsection{Simulation and design}

A schematic illustration of the high contrast transmitarray used for implementation of the wave plates is shown in Fig. 1. The transmitarray is composed of a periodic array of elliptical amorphous silicon (aSi) posts which are arranged in a triangular lattice, and located on a fused silica substrate. The lattice constant of the array is smaller than the desired operation wavelength, and the array does not diffract a normally incident beam into non-zero diffraction orders. Thus, the transmit array can be characterized by its transmission and reflection coefficients.

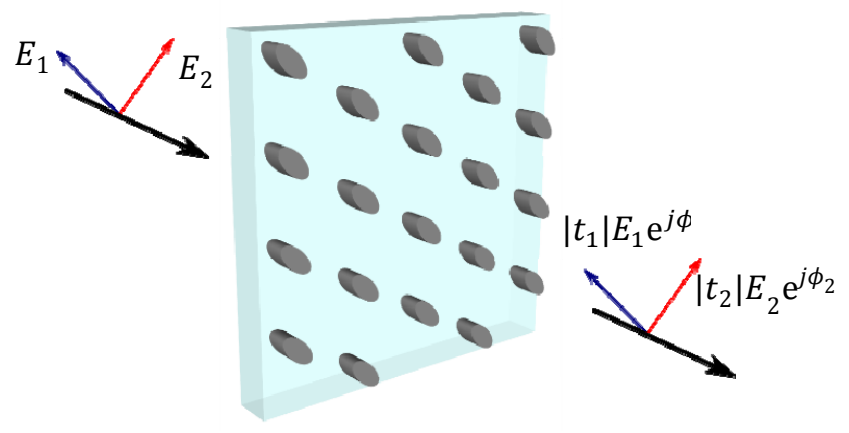

Figure 1. Schematic illustration of a high contrast transmitarray wave plate. The wave plate is composed of a periodic array of elliptical aSi silicon posts resting on a fused silica substrate. The array imposes different phase shifts to two normally incident optical waves linearly polarized along its two principle axes.

The ellipticity of the posts leads to different transmission coefficients for the two optical beams linearly polarized along the major and minor axes of the posts. Therefore, the transmitarray array shows an effective birefringence.

We used rigorous coupled wave analysis simulations and found the transmission coefficients of the transmitarray shown in Fig. 1 for different geometrical parameters. Simulation results shows that the fast and slow axes of the device are closely aligned along the minor and major axis of the elliptical posts, respectively. For operation wavelength of $\lambda=1550$ $\mathrm{nm}$, the simulation results showed that by choosing the post height of $1.23 \mu \mathrm{m}$ and the lattice constant of $1.1 \mu \mathrm{m}$, larger than $90 \%$ average transmission can be achieved for a wide range of ellipse diameters. The retardation of the transmitarray depends on the diameters of the elliptical posts and can be controlled by their proper selection. We realized a quarter wave plate by using elliptical posts with major and minor diameters of $360 \mathrm{~nm}$ and $315 \mathrm{~nm}$, and a half wave plate by using diameters of $405 \mathrm{~nm}$ and $285 \mathrm{~nm}$.

\subsection{Fabrication}

The wave plates were fabricated on a fused silica substrate. We deposited $1.23 \mu \mathrm{m}$ amorphous silicon on the substrate by using plasma enhanced chemical vapor deposition using a 5\% mixture of silane in argon. The refractive index of the deposited aSi was measured using a variable angle spectroscopic ellipsometer and was found as 3.40 at $\lambda=1550 \mathrm{~nm}$. The transmitarray patterns for the quarter and half wave plates (which are shown schematically in Fig. 2) where written on a positive resist using $100 \mathrm{kV}$ electron beam lithography. After the resist development, a $70 \mathrm{~nm}$ thick layer of aluminum oxide was deposited on the sample and was patterned by lifting up the resist in a solvent. The patterned aluminum oxide layer was used as a hard mask for etching the aSi layer in a mixture of $\mathrm{SF}_{6}$ and $\mathrm{C}_{4} \mathrm{~F}_{8}$ plasmas using inductively coupled plasma reactive ion etching. Subsequently, the aluminum oxide mask was etched in 1:1 mixture of ammonium hydroxide and hydrogen peroxide heated to $80^{\circ} \mathrm{C}$. Scanning electron microscope images of the fabricated devices are shown in Figs. 2 (a) and 2 (b). An optical micrograph of one of the fabricated device is shown in Fig. 1 (c) which shows the dimensions of the fabricated transmitarrays. 
(a)

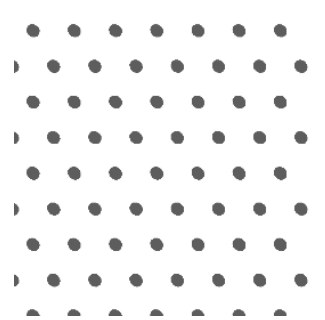

(b)

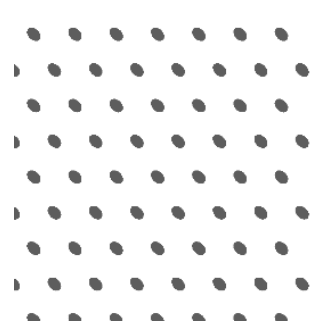

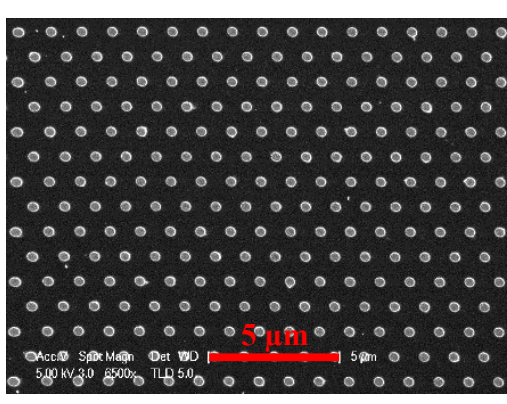
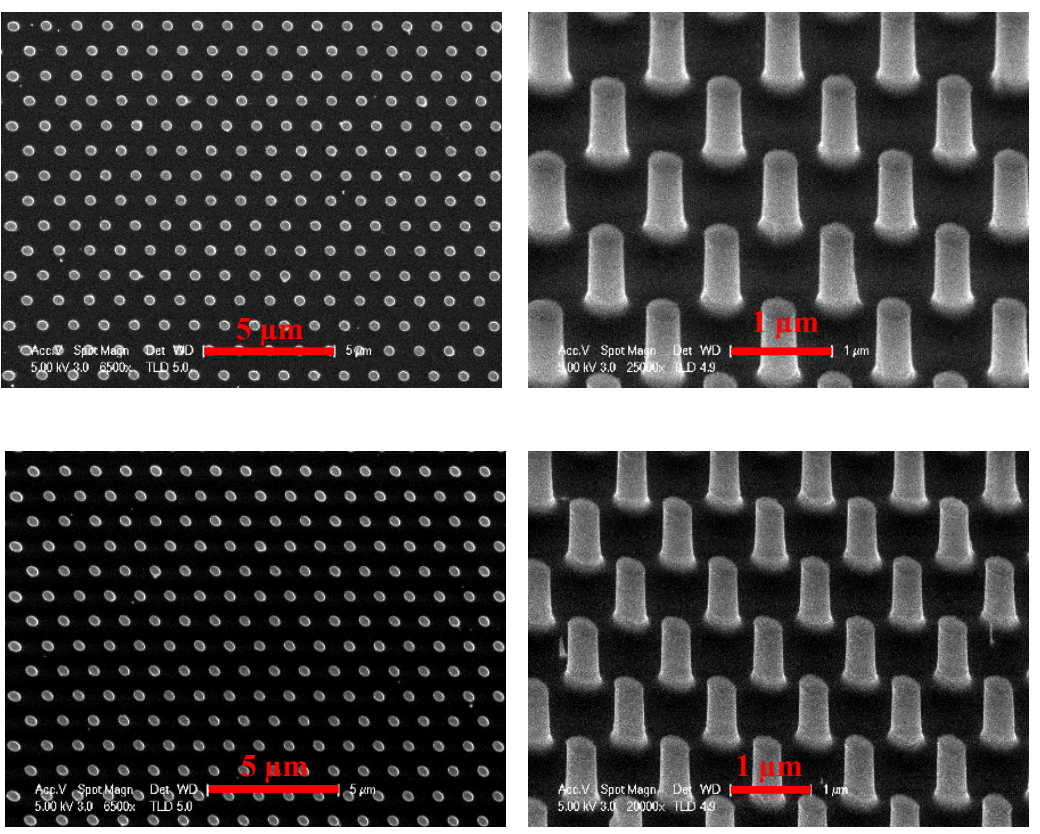

(c)

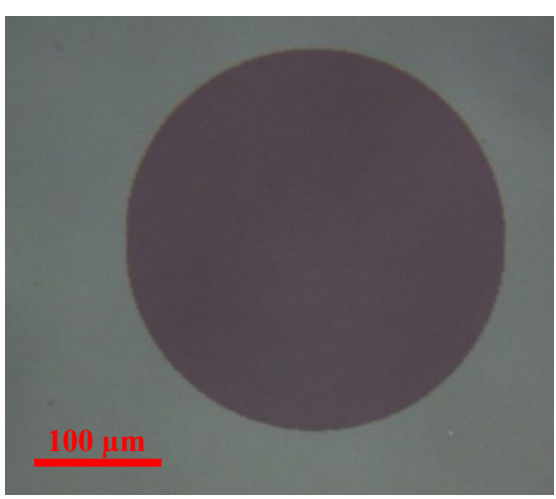

Figure 2. (a) Schematic illustration and scanning electron microscope images of a high contrast transmitarray quarter wave plate, and (b) a half wave plate. The lattice constant of the arrays are $1.1 \mu \mathrm{m}$, and the elliptical posts are $1.23 \mu \mathrm{m}$ tall. (c) Optical microscope image of a wave plate.

\subsection{Measurement}

The fabricated wave plates were characterized using the setup schematically shown in Fig. 3. Infrared light from a 1550 $\mathrm{nm}$ laser was passed through a polarization controller and coupled to free space using a fiber collimator. The output beam of the collimator was polarized after passing through the polarizer $\left(\mathrm{P}_{1}\right)$. The diameter of the collimated beam $(\sim 900$ $\mu \mathrm{m}$ ) was larger than the size of the fabricated wave plates (see Fig. 1(c)); therefore, a lens was used to focus the beam to a smaller spot. The light transmitted though the wave plate was collected using a 20X objective lens. Another polarizer $\left(\mathrm{P}_{2}\right)$ was placed after the objective lens and was used to analyze the polarization state of the transmitted light. After passing through the second polarizer, the light was passed through a tube lens which focused the light to a phosphorous coated CCD camera. The distance between the objective lens and the sample was adjusted such that the camera imaged the sample plane. The sample was mounted on an xyz stage and the camera image was used to align the wave plates and the incident beam. For optical power measurements, a flip mirror was inserted between the camera and the tube lens to direct the beam to a photodetector. The measured optical powers were normalized to the power measured when the sample was removed from the setup. 


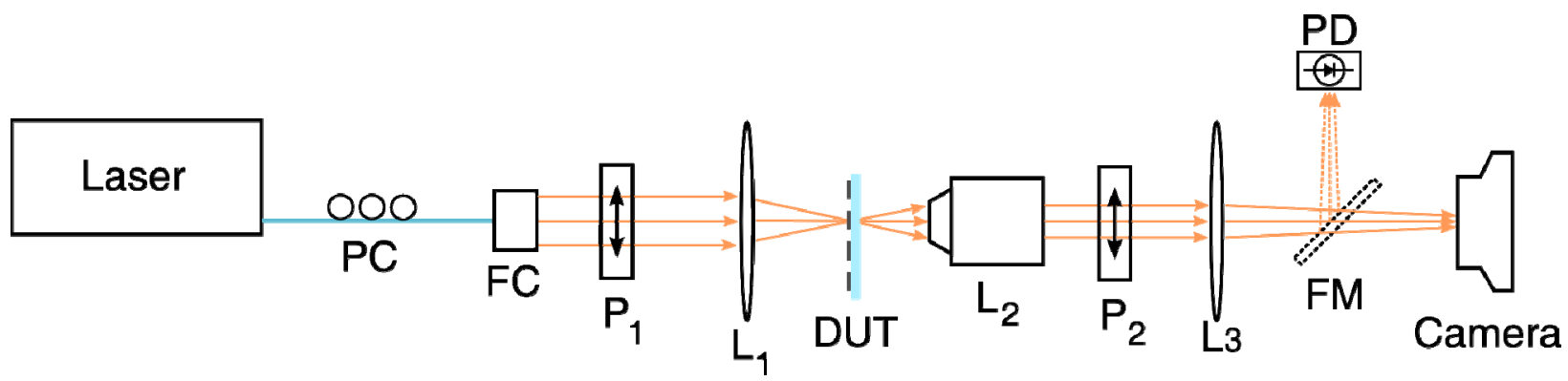

Figure 3. Schematic diagram of the measurement setup used for characterization of the wave plates and the vector beam generator. PC: polarization controller, FC: fiber collimator, P: polarizer, L: lens, DUT: device under test, FM: flip mirror, PD: photodetector.

To analyze the polarization state of the transmitted light, the power incident on the photodetector was measured as the transmission axes of the $\mathrm{P}_{1}$ and $\mathrm{P}_{2}$ polarizers were set to $0^{\circ}, 45^{\circ},-45^{\circ}$, and $90^{\circ}$. The measured data was fit to a Jones matrix model using the least squares fitting approach, and the transmission coefficients and the retardances of a quarter wave and a half wave plate were determined. The measurement results along with the design values for the diameters of the elliptical posts are presented in Table 1. The uncertainties in the measured quantities were computed using the Monte Carlo technique by taking into consideration the uncertainties in the optical power measurements. The more than $90 \%$ average transmissions and the accurate retardance values achieved using high contrast transmitarray wave plates make these devices viable candidates for practical applications. The smallest feature size of $\sim 300 \mathrm{~nm}$ allows for fabrication of larger wave plates at lower costs using conventional photolithography.

Table 1. Geometrical dimensions and measurement results of the fabricated wave plates.

\begin{tabular}{|l|l|l|l|l|l|}
\hline \multicolumn{1}{|c|}{ Device } & \multicolumn{1}{|c|}{$\mathbf{D}_{\mathbf{1}} \mathbf{( n m )}$} & \multicolumn{1}{|c|}{$\mathbf{D}_{\mathbf{2}} \mathbf{( n m )}$} & $\left|\mathbf{t}_{\mathbf{1}}\right|$ & $\left|\mathbf{t}_{\mathbf{2}}\right|$ & Retardance (waves) \\
\hline Quarter wave plate & 360 & 315 & $0.99 \pm 0.01$ & $0.90 \pm 0.01$ & $0.24 \pm 0.01$ \\
\hline Half wave plate & 405 & 285 & $0.95 \pm 0.02$ & $0.90 \pm 0.01$ & $0.5 \pm 0.02$ \\
\hline
\end{tabular}

\section{LOCAL POLARIZATION ROTATION USING SPATIALLY VARYING WAVE PLATES}

As we mentioned in the introduction section, the high refractive index contrast between the aSi posts and their surroundings leads to weak coupling among them, and the light scattered by each post is not significantly affected by the shape and orientations of its neighboring posts. As a result, gradual modification of the rotation axes of the posts (as schematically shown in Fig. 4) does not significantly affect the local transmission coefficient of the array. An example of a device which is created by gradual rotation of the fast axis of a half wave plate is shown in Fig. 4 . The device is composed of an array of similar elliptical aSi posts with the same dimensions as the posts we used for implementation of the half wave plates $\left(D_{1}=405 \mathrm{~nm}\right.$ and $\left.D_{2}=285 \mathrm{~nm}\right)$. The elliptical posts are rotated by different angles such that the fast axis of an elliptical post located at polar coordinate $(r, \theta)$ is aligned along the $\theta / 2$ direction; therefore, the polarization of an X-polarized incident beam will be rotated at the position of the post by $\theta$ and the output beam will be radially polarized. Similarly, polarization direction of a y-polarized beam will be locally rotated by $-\theta$, and the output beam will be azimuthally polarized. It should be noted that for linear incident beams the relative phase of light passed through different points of the device is not modified and the device does not change the beam's wavefront. On the other hand, a circularly polarized beam undergoes polarization direction reversal and a local geometric phase shift equal to $2 \theta$ [9].

The device shown schematically sown in Fig. 4 was fabricated using the same process we used for fabrication of the wave plates. Scanning electron microscope images of the device are also shown in Fig. 4. 

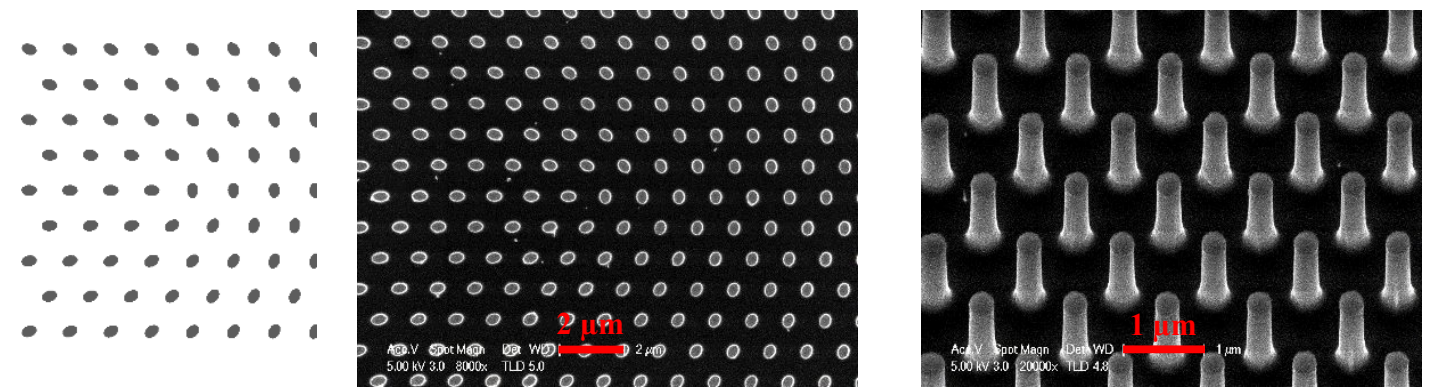

Figure 4. Schematic illustration and scanning electron microscope images of a spatially varying high contrast transmit array half wave plate which converts linearly polarized incident beams to radially and azimuthally polarized beams.
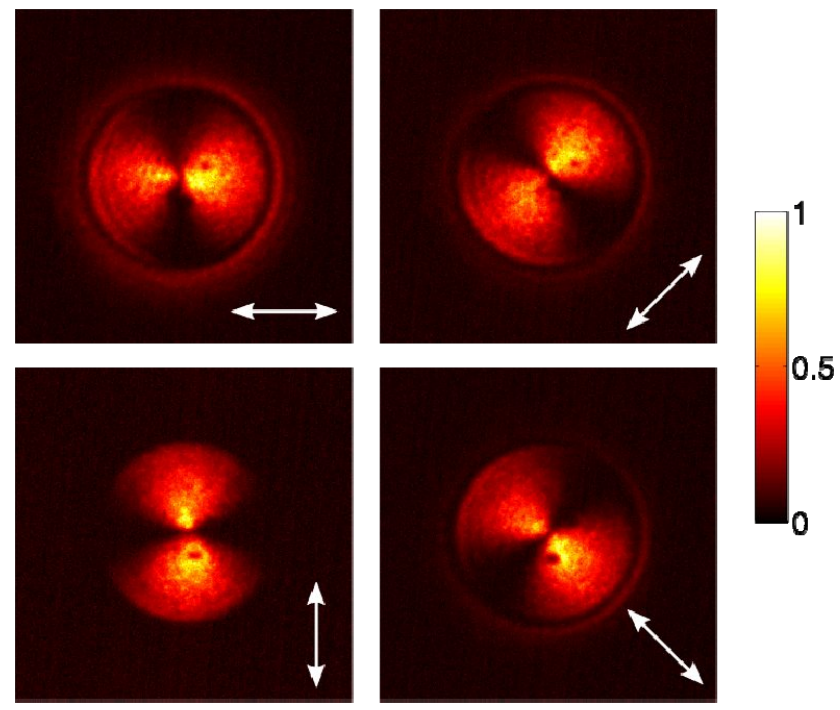

$200 \mu \mathrm{m}$

Figure 5. Measured intensity profiles of the light transmitted through a spatially varying high contrast transmitarray half wave plate which converts x-polarized polarized incident beams to a radially polarized beam. The double sided white arrows shown on the lower right corner of the images represent the transmission axis of the polarizer $\mathrm{P}_{2}$ shown in Fig. 3.

We characterized the device using the measurement setup shown in Fig. 3. Figure 5 shows the optical intensity profiles captured using the camera when the incident beam was polarized along the horizontal direction and the transmission axis of the polarizer $\mathrm{P}_{2}$ was set along four different directions shown by the white arrows. The rotation of the recorded intensity profile along with the transmission angle of the polarizer verifies the radial polarization of the output beam. The non-uniformities observed in the measured intensity profiles are due to the phosphorous coating on the camera and were observed even when the device was removed from the setup. The transmission efficiency of $84 \pm 2 \%$ for the device was obtained by removing the polarizer $\mathrm{P}_{2}$ from the setup, directing the transmitted light to the photodetector using the flip mirror, and dividing the measured power to the power measured when the device was removed from the setup. 


\section{CONCLUSION}

We demonstrated highly efficient wave plates implemented using a high contrast transmitarray platform based on elliptical aSi posts on glass. We showed that high transmission and different retardances can be achieved using fixed aSi thickness and with different post diameters. The measured average transmission efficiency of $\sim 90 \%$, the subwavelength thickness of the device, the relative simplicity of the fabrication process, and the possibility of fabrication of these wave plates over large areas using photolithography make them attractive for on chip and free space optics applications. One of the main advantages of the high contrast transmit arrays is the weak coupling among the elements in the array which provides the possibility of realization of devices with spatially varying properties. By design and fabrication a radial/ azimuthal polarizer, we experimentally demonstrated that local polarization rotation can be achieved by using a half wave plate whose fast axis is gradually rotated. We should note that the devices we presented here are expected to have narrower bandwidth compared to conventional birefringent crystal based wave plates. Further improvement of the bandwidth might be achievable by optimizing the geometrical parameters of the high contrast transmitarray.

\section{REFERENCES}

[1] M. Mutlu, A. E. Akosman, G. Kurt et al., "Experimental realization of a high-contrast grating based broadband quarter-wave plate," Opt Express, 20(25), 27966-73 (2012).

[2] Y. Yang, W. Wang, P. Moitra et al., "Dielectric meta-reflectarray for broadband linear polarization conversion and optical vortex generation," Nano Lett, 14(3), 1394-9 (2014).

[3] Y. Zhao, and A. Alu, "Manipulating light polarization with ultrathin plasmonic metasurfaces," Physical Review B, 84(20), 205428 (2011).

[4] E. B. Grann, M. G. Moharam, and D. A. Pommet, "Artificial uniaxial and biaxial dielectrics with use of twodimensional subwavelength binary gratings," Journal of the Optical Society of America A, 11(10), 2695-2703 (1994).

[5] D. C. Flanders, "Submicrometer periodicity gratings as artificial anisotropic dielectrics," Applied Physics Letters, 42(6), 492-494 (1983).

[6] L. Huang, X. Chen, H. Mühlenbernd et al., "Three-dimensional optical holography using a plasmonic metasurface," Nature communications, 4, (2013).

[7] D. Lin, P. Fan, E. Hasman et al., "Dielectric gradient metasurface optical elements," science, 345(6194), 298302 (2014).

[8] M. Khorasaninejad, and K. B. Crozier, "Separating Left- from Right-Circularly Polarized Light with a Dielectric Metamaterial," OSA Technical Digest (online). SF1O.2.

[9] M. V. Berry, "The Adiabatic Phase and Pancharatnam's Phase for Polarized Light," Journal of Modern Optics, 34(11), 1401-1407 (1987).

[10] A. Arbabi, and A. Faraon, "Fundamental Limits of Ultrathin Metasurfaces," arXiv preprint arXiv:1411.2537, (2014).

[11] F. Aieta, P. Genevet, M. A. Kats et al., "Aberration-free ultrathin flat lenses and axicons at telecom wavelengths based on plasmonic metasurfaces," Nano letters, 12(9), $4932-4936$ (2012).

[12] A. Arbabi, Y. Horie, A. J. Ball et al., "Subwavelength-thick Lenses with High Numerical Apertures and Large Efficiency Based on High Contrast Transmitarrays," arXiv preprint arXiv:1410.8261, (2014).

[13] A. Arbabi, M. Bagheri, A. J. Ball et al., "Controlling the Phase Front of Optical Fiber Beams using High Contrast Metastructures." STu3M. 4. 